\title{
OPINIÓN
}

\section{APUNTES ACERCA DE LA UNIVERSIDAD EN TIEMPOS DE CONFLICTO*}

\author{
Enrique Barros \\ Universidad de Chile \\ Arturo Fontaine \\ Universidad de Chile
}

\begin{abstract}
Resumen: En este ensayo los autores sostienen que es necesario distinguir entre la finalidad de la educación como tal, que es un fin en sí misma, y sus efectos colaterales, como el aumento en la productividad. Afirman que el cultivo de las ciencias y humanidades incide directamente en la calidad del profesorado escolar. Critican la existencia en Chile de universidades con fines de lucro encubierto que vulneran el espíritu de la ley que lo prohíbe. La norma quiere evitar el conflicto de interés entre el cumplimiento de metas estrictamente académicas y las típicas de una empresa comercial. Las limitaciones e imperfecciones propias de los instrumentos de medición de la educación, así como sus efectos no buscados, hacen más realista, a juicio de los autores, establecer reglas
\end{abstract}

EnRIQUe Barros. Abogado, profesor de la Facultad de Derecho de la Universidad de Chile y miembro del Consejo Directivo del Centro de Estudios Públicos (ebarros@blc.cl).

Arturo Fontaine. Profesor del Departamento de Filosofía de la Facultad de Filosofía y Humanidades de la Universidad de Chile y director del Centro de Estudios Públicos (afontaine@cepchile.cl).

* Versión extendida del texto que apareció en El Mercurio de Santiago el 24 de julio de 2011.

Estudios Públicos, 124 (primavera 2011). 
generales y simples de carácter prudencial para evitar dichos conflictos de interés.

Palabras clave: educación, mediciones educacionales, universidad, universidades con fines de lucro, conflicto universitario chileno, Universidad de Chile.

Recibido y aceptado: diciembre 2011.

\title{
NOTES ON THE UNIVERSITY IN TIMES OF CONFLICT
}

\begin{abstract}
It is argued in this essay that the aim of education as an end in itself must be distinguished from the side effects of education such as growth in productivity. The authors contend that the cultivation of the sciences and humanities has a direct bearing on the quality of school teachers.

According to them, there are universities in Chile that have a concealed profit-making purpose and consequently violate the spirit of the law. The Chilean norm, which expressly forbids the universities to have profit-making ends, intends to avoid a conflict of interests between the fulfilment of strict academic goals and the typical goals of a commercial enterprise. The limitations and imperfections of the instruments of educational measurement, as well as their non intended effects, make more realistic - the authors say- to establish general and simple rules of good judgment in order to avoid such clash of interests.
\end{abstract}

Keywords: education, educational measurements, universities, universities with profit-making purposes, Chilean university conflict, University of Chile.

Received and accepted: December 2011.

E

erróneo concebir la educación como un medio para mejorar la productividad y contribuir al crecimiento económico. Una sociedad con muchos enfermos es menos productiva que una con su población sana. Sin embargo, la salud es un fin en sí misma, aunque también sea valiosa por su aporte a la productividad. Algo análogo ocurre con la educación. Los constructores de la biblioteca de Alejandría, los monjes medievales que conservaron los folios de los clásicos, los humanistas que leyeron esos textos y con ello, de algún modo, pusieron en marcha el mundo moderno no lo hicieron para lograr un beneficio ajeno al bien que por sí mismo representaba el saber y entender. 
¿Qué movió a Darwin a plantearse sus reflexiones observando la fauna de las Islas Galápagos? ¿Por qué nos atrae seguir su modo de razonar? Simplemente porque nuestra natural curiosidad, que ha sido el gran motor del desarrollo de la especie, adquiere en la ciencia por sí misma una justificación. Por cierto que la educación tiene como un efecto colateral la mejoría de la productividad, pero esa no es su finalidad. La educación nos importa porque en ella se pone en juego la humanidad de lo humano. Cuando un niño pregunta de donde nacen las estrellas es movido por el asombro que, dice Aristóteles, está en el origen del conocimiento. El estado debe apostar a lo que nos hace mejores seres humanos, porque es a partir de allí que cobran sentido nuestra ciudadanía democrática y contenido las oportunidades que ofrece el progreso material.

La universidad es el lugar de ese asombro primordial, sin el cual no hay amor al conocimiento. La creación y transmisión del conocimiento en su más alto nivel da sentido a la comunidad universitaria. Por eso, el cultivo de las matemáticas, las ciencias, las humanidades deben estar en el centro de su actividad. Es el estudio de esas disciplinas matrices lo que luego permite, por ejemplo, tener profesores de calidad en los liceos y colegios, es decir, profesores capaces de inquietar, de abrir mentes y orientar personas. Por cierto que esta tarea es cumplida con éxito si el propio profesor ha llegado a entender los límites de su ciencia y está en cierto sentido maravillado por su entendimiento. En última instancia, el nivel de los departamentos de matemáticas de la universidad, que no persiguen en sí mismos fin instrumental alguno, determina el nivel de los profesores de matemáticas de la media y la básica; y, en cascada, el de gran parte de nuestros profesionales.

Un profesor enseña porque intuye que entender, por ejemplo las leyes de la óptica, es una experiencia fascinante en sí misma, como contemplar un paisaje de singular belleza o ver un gran partido de tenis o escuchar una sonata de Mozart. Lo atractivo de ser profesor es ayudar a entender. Eso hacemos los profesores en las clases. Esto no es un sueño romántico, como le dijo a uno de nosotros hace unas semanas un experto, quien, por cierto, no hace clases. Es lo que nos ocurre a diario. Que ello cause sorpresa y escepticismo en el experto indica hasta qué punto es incomprendida hoy en Chile la profesión docente. El principio es el mismo en las ciencias teóricas que en los saberes prácticos. Porque entiende cómo funciona el motor, el buen mecánico es capaz de pillar la pana y repararla. Gracias a que entiende las coordenadas de su discipli- 
na, el buen profesional es capaz de ganarse la vida prestando servicios en eso que llegó a entender. Y, claro, alguien lo hizo posible. Alguien lo condujo de entendimiento en entendimiento hasta que llegó a ser un "entendido".

No es cierto que los beneficios de la universidad se agoten en los que asisten y se gradúan en ella. La educación tiene un efecto expansivo que alcanza a todos y, en especial, a los que vienen de hogares de escasa tradición cultural y encuentran en la escuela la mejor oportunidad de dar un salto cualitativo respecto de la anterior generación. El interés por conocer se contagia desde la universidad y desde allí por mil vasos comunicantes - la prensa entre ellos - se reparte por el cuerpo social. A su vez, la cultura que nace fuera de la universidad fluye en ella (como se muestra en los cambios de intereses de cada generación de estudiantes). Los conflictos universitarios graves llegan a La Moneda porque, consciente o inconscientemente, partimos de la base de que lo que ocurre en la universidad, tarde o temprano, nos atañe a todos.

Quizás haya que aceptar que sólo algunas universidades chilenas están en condiciones, por ahora, de cumplir cabalmente esa misión. Eso obliga a reconocer, entonces, que nuestra investigación y enseñanza dependen de las pocas universidades que por su tradición o universalidad, por su vocación académica y de excelencia, se acercan a la misión de un gran centro de estudios superiores. Esas universidades son el pulmón que oxigena todo el sistema. Esas universidades deben atraer a las mejores cabezas que tengan vocación por el estudio y comprometerlas en una vida dedicada al conocimiento y al desarrollo de las sensibilidades superiores que caracterizan a una persona cultivada. Chile necesita que los jóvenes más dotados e inclinados a investigar y enseñar puedan consagrarse a esa tarea con entusiasmo y sin zozobras. Para ello, se debe asegurar un financiamiento estable que lo haga posible. De lo contrario, miles de jóvenes graduados en grandes universidades extranjeras, gracias al programa Becas Chile, terminarán trabajando en universidades fuera de Chile o irán al mercado profesional, a pesar de sus vocaciones académicas.

\section{Universidades con fines de lucro y conflictos de interés}

Las que reconocemos como las mejores universidades se financian fundamentalmente con aportes del Estado o una combinación del Estado más cuantiosos fondos obtenidos de donaciones privadas. El 
aporte que hacen las familias, vía matrícula diferenciada, alcanza en una universidad como Harvard, sólo al 19 por ciento de su presupuesto anual, según datos del año pasado. En cambio, los intereses de su fondo de inversiones representan un 35 por ciento. Otras donaciones aportan un 7 por ciento adicional. El Estado aporta un 17 por ciento. Harvard - como tantas de las mejores universidades del mundo- es una corporación privada sin fines de lucro y el grueso de su presupuesto proviene de donaciones. Por eso, es difícil que un joven verdaderamente excepcional quede fuera de las más grandes universidades porque carece de recursos.

Pareciera que en Chile están en deuda tanto el Estado como la empresa. Pareciera que el paro prolongado, ha logrado un grado de coincidencia en esto. El Estado ha fallado porque su aporte es bajo y su sistema de créditos requiere cirugía mayor. Y las universidades con lucro encubierto han sembrado la duda y la desconfianza. A su vez, ese smog que se ha levantado en torno a la educación superior privada también oscurece el creciente aporte que están realizando universidades privadas sin fines de lucro que progresan y mejoran día a día.

La ley obliga a las universidades a constituirse como corporaciones o fundaciones, esto es, como entidades sin fines de lucro. La norma quiere evitar el conflicto de interés entre el cumplimiento de metas estrictamente académicas y las típicas de una empresa comercial; por ejemplo, entre la conveniencia de construir un laboratorio de física o de idiomas y la expectativa de los dueños de una empresa de servicios de extraer las utilidades que deja la operación. Los caminos indirectos para obtener las rentas por otros caminos son igualmente ajenos al fin de esa norma. Por eso, si quienes gobiernan la universidad retiran dividendos vinculados a su gestión, tal conflicto reaparecería y la norma que prohíbe el lucro carecería de razón de ser. La norma, que es preventiva y prudencial, quedaría en tal caso sin efecto gracias a una martingala.

Los conflictos potenciales son diversos. El más evidente apunta al número de alumnos. La calidad de la enseñanza muchas veces mejora con cursos más bien pequeños. Pero si la universidad es un negocio, por obvias economías de escala convendrá hacer lo contrario, es decir, aumentar al máximo el número de alumnos por profesor. Tampoco convendrá tener profesores a dedicados al estudio e investigación y con tiempo para corregir con esmero y formar a sus alumnos. Y para enseñar no se optará por expertos excepcionales, que tienen alternati- 
vas promisorias en su vida académica o profesional, sino más bien por secundones que se pasean repitiendo una letanía de aula en aula. La universidad se llenará de instructores mediocres (que serán llamados profesores) que hacen clases como podrían hacer cualquiera otra tarea.

La investigación en ciencias básicas rara vez es un negocio para un inversionista, aunque indirectamente produzca beneficios económicos a la sociedad. En Estados Unidos el empresariado apostó tempranamente a la libertad de la cultura y al valor de las ciencias. Las generosas donaciones que hacen las empresas sostienen un sistema universitario mixto, estatal y privado, sin fines de lucro, que se sitúa hoy en la vanguardia del conocimiento en muchísimos campos. Esta es una importantísima fuente de legitimación del capitalismo. En Chile, por el contrario, las martingalas contribuyen a deslegitimarlo.

Ya Tocqueville observó la significación que tenían las asociaciones voluntarias sin fines de lucro en los Estados Unidos. La National Gallery, por ejemplo, es una donación de la familia Mellon; y la Universidad de Chicago fue creada por los Rockefeller. Con los años, ese rasgo cultural no ha hecho más que crecer y multiplicarse. La cohesión social y la confianza en la libertad de las personas se fortalecen con la iniciativa y la capacidad para asociarse en tareas de interés general. En Chile carecemos de esta fuerte tradición de que fines públicos sean asumidos desde dentro de la sociedad civil. Así y todo, las donaciones a las universidades han ido aumentando. Eso es muy esperanzador. Sin embargo, los montos que se donan no guardan relación directa con la calidad académica de la institución. Así, la Universidad de Chile, pese a tener el mayor número de publicaciones en revistas especializadas internacionales, no es la que recibe más donaciones. Una vocación por la excelencia es parte de un cambio cultural más profundo, que vea en el desarrollo de la universidad al más alto nivel un camino para hacer más inteligente, culto y fuerte a nuestro país.

\section{El difícil tema de las mediciones de calidad}

Un estudiante, por serlo, es un evaluador sumamente imperfecto de la calidad de la docencia que recibe en su universidad. (¿Cuántos semestres de cálculo necesita un administrador de empresas? ¿Dos, uno, ninguno?) Cuando el joven entra a trabajar y cae en la cuenta de sus falencias, ya es tarde (y muy caro) para empezar a estudiar de nuevo. 
No ocurre lo mismo en el mercado de las camisas o de las corbatas. El problema con los argumentos por analogía es saber hasta dónde llevar la similitud y dónde marcar la diferencia. Si se parte por las semejanzas, es evidente que el mercado universitario chileno no pasa la prueba del consentimiento informado, que trata de cautelar el derecho en los contratos con consumidores. Muchos de los jóvenes más desventajados (y sus familias) toman decisiones cruciales respecto de su educación tras el velo más denso de desinformación.

Nuestro sistema universitario no asegura que los licenciados de las universidades más mediocres tengan las habilidades y conocimientos suficientes para desempeñarse en sus profesiones. Sin embargo, esos grados les permiten obtener sin más la calificación profesional. El resultado es que se ve comprometida la confianza pública en las capacidades asociadas a las profesiones. De hecho, a pesar de sus limitaciones, es probable que se requiera un sistema público de controles externos para comprobar que los egresados de las universidades tengan las aptitudes para desempeñarse como abogados, arquitectos, constructores o ingenieros (los médicos ya han avanzado en esta dirección gracias a regulaciones que rigen el acceso a las especialidades).

En verdad, resulta más fácil medir destrezas profesionales que la calidad de la educación. De hecho, las mejores universidades no persiguen sólo desarrollar destrezas profesionales concretas. Los indicadores y las acreditaciones ayudan y son muy útiles, pero deben administrarse con sentido de sus limitaciones. No son la panacea que solucionará todas las fallas de la educación básica, media y superior como a veces pareciera creerse. Por la compleja y sutil multidimensionalidad de los procesos educativos, las mediciones son siempre parciales. A menudo las evaluaciones pueden ser sorteadas y desvirtuadas desnaturalizando la docencia hasta hacer de ella un mero adiestramiento para rendir una prueba. Es decir, la medición puede alterar lo que se busca medir.

Además, hay fuertes indicios de que, por ejemplo, la empleabilidad y el nivel de ingreso de un graduado chileno tiene más que ver con su medio social que con sus notas. En consecuencia, es probable ese indicador premie a las universidades que capturen a jóvenes de mejor situación económica y buenas redes.

Por otro lado, existen grandes diferencias entre los escasos recursos asignados a difundir información objetiva y las millonarias campañas publicitarias de ciertas universidades. Tampoco están claras 
la naturaleza y calidad de la información requerida. Los costos de informarse bien son elevados para un joven dieciocho años. Y si se atiende a que usualmente quienes disfrutan de menor capital cultural tienen proporcionalmente menos probabilidades de acceso a las universidades reconocidas como las mejores, la decisión de invertir parte del patrimonio familiar para que el hijo sea universitario se suele tomar a tientas.

En esta cuestión tan delicada, como es qué estudiar y dónde hacerlo, nada hay más ciego a la realidad que suponer una racionalidad económica perfecta. Ese es un supuesto utópico. Es cierto que, con ciertas distorsiones, en general la experiencia muestra cuales son las mejores universidades, públicas y privadas. Pero no hay información homologable que permita saber cuáles simplemente no dan la marca. En definitiva, aunque es obvio que resulta más compleja la decisión por una carrera y una universidad que la de comprar unas zapatillas, se protege mejor como consumidor al que compra un par de zapatillas; poco se ha hecho por años para proteger al estudiante y a su familia cuando se toma una decisión tanto más relevante.

Más importante: aspectos importantísimos de la educación —quizás los más importantes para la formación de un joven- no admiten mediciones cuantitativas. El año pasado en el CEP, James Heckman, Premio Nobel de economía, expuso un trabajo que indica que las habilidades que más remunera el mercado del trabajo son las "habilidades blandas", las vinculadas al carácter, y no los conocimientos duros, como por ejemplo las matemáticas (ver www.cepchile.cl video y láminas "James Heckman en el CEP”). El énfasis excesivo en lo medible pone el foco de la educación en eso, en lo medido, en desmedro, muchas veces, de actividades más valiosas y que no se miden.

Quienes favorecen la creación de universidades con fines de lucro responden al argumento del conflicto de interés apelando a la información y las mediciones que debería llevar a cabo el Estado. Ahora bien, si la institución bajo evaluación tiene fines de lucro, los incentivos para eludirla o tergiversarla serán mayores. Toda una alambicada parafernalia de controles y evaluaciones de cientos de carreras pasaría a ser responsabilidad del Estado y, en la práctica, de funcionarios públicos que actúan en una estructura que no tiene fines de lucro. Los defensores a ultranza de las empresas comerciales de educación superior, al revés de lo que dicen, terminan depositando enorme confianza en el Estado, en sus regulaciones y funcionarios. Si son sinceros. 
Esto no impide, a nuestro juicio, que en el futuro pudieran permitirse universidades organizadas como empresas de servicios, que deberían pagar impuestos como cualquier empresa y no podrían recibir, claro, donaciones con los beneficios tributarios de las que persiguen fines propiamente universitarios. Por prudencia, dichas universidades tampoco deberían recibir aportes del Estado. Los conflictos de interés ya mencionados aconsejan extrema cautela. En Estados Unidos estas universidades existen y hasta ahora se han orientado a una formación puramente instrumental, incluso en disciplinas que tienen una tradición intelectual o científica fuerte, como el derecho y las ingenierías. En Brasil llenan un vacío que en países con un capitalismo más maduro llenan el Estado e instituciones educacionales sin fines de lucro. Estas universidades con fines de lucro, con todos los resguardos de información, pueden ser efectivas como instancias educativas de formación técnica más que universitaria. Es un dicho conocido en Estados Unidos que estas universidades forman profesionales de 'Main Street' (el nombre que suele tener la calle que atraviesa los pueblos pequeños), esto es, profesionales habilitados para solucionar problemas cotidianos del vecindario. Pero, así y todo, ninguno de los licenciados puede llamarse abogado si antes no ha pasado un examen general de habilidades profesionales básicas.

\section{EI Presidente es el patrono de la Universidad de Chile}

Las concordancias preliminares a que se ha llegado durante el conflicto que estamos viviendo indican que el gobierno reconoce que el aporte del Estado a la educación es insuficiente en nuestro actual grado de desarrollo. Hay aquí una oportunidad para que también la empresa privada, dado este diagnóstico común, refuerce sus donaciones a entidades propiamente universitarias, impulsando a nuestras universidades de mayor excelencia académica, que hoy representan menos del $2 \%$ del presupuesto fiscal (El Mercurio, 17 de julio 2011, B 4). La manera de responder al descrédito del negocio de educación superior es mostrar un empresariado generoso y con fe en las de la libertad de la cultura.

Una de las palancas del crecimiento económico de la India es el desarrollo de la informática que se concentra en Bangalore, el Silicon Valley de la India. Ese fenómeno se explica, en parte, como una 
consecuencia indirecta, y en su momento no pensada, de los institutos tecnológicos de alto nivel, los Indian Institutes of Technology (IIT), universidades estilo MIT, que se crearon en tiempos de Nehru. La apertura de la economía y los nuevos desarrollos de la ciencia de la computación han valorado ese esfuerzo. Son universidades estatales muy selectivas. Aceptan sólo al 2 por ciento de los que postulan (Harvard acepta al 10 por ciento) Una de las empresas más exitosas en el rubro de la informática es Infosys. Su fundador y CEO hasta el 2001, Narayan Murthy, uno de los empresarios más famosos de la India, es uno de los principales donantes de IIT, donde obtuvo su master (pese a ello su hijo, por no alcanzar los puntajes requeridos, no fue aceptado por ningún IIT y estudió en la Universidad de Cornell). Desde su retiro, asumió la presidencia del directorio del Indian Institute of Information Technology de Bangalore. "El poder del dinero es el poder de dar", ha dicho Murthy. Ese es el tipo de relación que se establece en las economías modernas entre empresa y universidad.

El Presidente de Chile es el patrono de la Universidad de Chile. A veces, como profesor, uno no siente ese afecto en las palabras y gestos del Presidente; ese cuidado de la institución que es propio de un patrono. Tampoco lo sentimos, la verdad, en los presidentes anteriores. Es cierto que recíprocamente la Universidad de Chile tiene la responsabilidad de darse un buen gobierno, tarea que es dificultada por una burocracia y por un corporativismo que la asfixian, porque la mera concurrencia de intereses particulares hace que en su gobierno a menudo se oscurezca el horizonte de la excelencia académica, que al final de cuentas es su única meta legítima. Pero, aun con sus dificultades, la Universidad de Chile es un faro de la cultura chilena, porque en ella late, al menos por aquí y por allá, el espíritu libre y sensible que respira una universidad de excelencia.

La universidad aspira a que en nuestros estudiantes se encarne una tradición cultural, que ha de entenderse como una larga conversación entre una muy larga secuencia de generaciones. Como toda buena conversación hay en ella momentos mejores que otros, mutaciones y contradicciones. Pero es siempre al interior de esa conversación abierta que surge y fluye el tú, el yo, el nosotros. Esa conversación que nos constituye como seres humanos, lo sepamos o no, adquiere una particular densidad en la vida al interior de la universidad. No podemos 
examinar nuestra cultura desde un punto de vista enteramente exterior a ella. En el curso de ese río descubrimos la amistad, las fallas morales y las virtudes, el abuso y la justicia, nuestros miedos e incertidumbres, la belleza del conocimiento, y hasta las formas del amor y el sentido del humor. Y presentimos el tamaño de nuestra ignorancia. 